\title{
Aurore boreali \\ osservate dalla stazione astronomica di Loiano \\ negli anni 1957-1958
}

\author{
P. MAFFEI
}

Negli anni 1957 e 1958 tre aurore polari venivano occasionalmente osservate dalla Stazione dell'Osservatorio Astronomico Universitario di Bologna situata nei pressi di Loiano. L'eccezionalità del fenomeno per la nostra latitudine ci induceva a compiere osservazioni visuali, sia pure sommarie, nonostante ciò esulasse completamente dall'indirizzo delle nostre ricerche.

Tutte le osservazioni venivano compiute dalla Stazione stessa avente le seguenti coordinate geografiche:

$$
\varphi=+44015^{\prime} 23^{\prime \prime} ; \lambda=0^{\mathrm{h}} 45^{\mathrm{m}} 20^{\text {s }} \text { Est di Greenw.; } \mathrm{h}=800 \mathrm{~m}
$$

Gli istanti sono espressi in T. U. Le misure espresse in gradi sono state compiute empiricamente a stima e risultano, semmai, approssimate per difetto. Per la prima aurora le descrizioni che seguono venivano scritte nel corso del fenomeno; per le altre due sono state registrate mediante un magnetofono. Questo secondo metodo si è dimostrato particolarmente efficace in questo genere di osservazioni, evitando perdite di tempo e distrazioni e dando all'osservatore la possibilità di mantenersi nel buio più completo, in modo da seguire ed annotare anche le luminosità più deboli e le variazioni più rapide.

Delle tre aurore la più imponente è stata certamente quella del 21 gennaio 1957 , specialmente nella sua fase oltre le ore 22 . Pure notevole l'aurora del 4 settembre 1958 quantunque osservata nelle più avverse condizioni: all'inizio dietro una cortina di fitti veli, dopo le ore 21 con la Luna sempre più alta sull'orizzonte.

\section{1-22 GenNaIo 1957.}

Il fenomeno veniva notato a $20^{\mathrm{ln}} 02^{\mathrm{m}}$ (T. U.), nel corso delle consuete osservazioni astronomiche, mentre la cupola era volta verso Sud. Da 
quel momento ne veniva iniziata l'osservazione, favorita da un cielo limpido e completamente privo di nubi. Diamo, qui di seguito, la descrizione delle varie fasi. La vistosità del fenomeno viene indicata con un punto esclamativo a sinistra dell'ora di osservazione.

!! $20^{\mathrm{h}} 02^{\mathrm{m}}$ la luce aurorale si estende per circa $45^{\circ}$ a partire dalla zona della costellazione del Cigno, immersa in una luce rossastra sfumante nel rosa e nel verde verso Nord. Bei raggi giallastri appaiono e scompaiono rapidamente, particolarmente nella zona di massima intensità. Degno di nota uno di circa $20^{\circ}$ di altezza, apparso intorno alla fine $\left(20^{\mathrm{h}} 05^{\mathrm{n}}\right)$ di questa fase.

2010 la luce aurorale è pressochè scomparsa.

! 2018 riappare una zona luminosa, ampia circa $25^{\circ}$, centrata esattamente a Nord.

!! 2025 compaiono due intensi raggi larghi circa $5^{\circ}$ e alti circa $20^{\circ}$.

2029 rimane una zona rossastra di $20^{\circ}$ di ampiezza in corrispondenza della costellazione del Cigno.

2034 compaiono due intensi e larghi raggi rossastri nella zona del Cigno.

2040 nessuna traccia di luce aurorale.

2044 - 2046 leggerissime luminosità appaiono e scompaiono nella zona Nord.

2050 un leggerissimo raggio appare e svanisce nella zona Nord.

2055 leggeri raggi appaiono e scompaiono nella zona Nord.

! 2102 leggeri raggi.

! 2105 riappare, leggera, la colorazione rossastra.

2112 leggerissima luce rosa nella zona del Cigno. Appare e scompare un raggio.

2122 su tutto l'orizzonte settentrionale, per una lunghezza di circa $45^{\circ}$, si estende una debolissima luce rossa.

! 2124 intensi e larghi raggi nella zona $\mathrm{N}-\mathrm{NW}$, dove da poco è tramontata la costellazione del Cigno.

2135 ancora alcuni deboli raggi.

2145 zona rossastra a N-NW. 
!!!!! $22^{\mathrm{h}} 02^{\mathrm{m}}$ appaiono numerosi e intensi raggi giallastri con sfumature verde pallido su fondo unito rosa-rosso, disposti in uno splendido arco di circa $100^{\circ}$ di lunghezza e $25^{\circ}$ di spessore. L'arco si innalza al di sopra dell'orizzonte come uno splendido ponte luminoso; al di sotto di questo una zona, limitata alla base dall'orizzonte, splende di intensa luce bianca verdastra.

!!! 2211 i raggi svaniscono.

!!!! 2212 i raggi riappaiono

!!!! 2220 tutto l'arco tende a svanire e restringersi; i colori si fanno di un rosso sempre più cupo.

!!! 2225 splendidi larghi raggi nella zona $\mathrm{N}-\mathrm{NW}$.

!!! 2230 due parti dell'arco rimangono intense, rosso cupo, solcate a momenti da qualche raggio. Una a Nord, leggermente spostata ad Est e un'altra nella zona NW.

!! 2235 le due zone continuano ad essere visibili ma sempre più deboli e di un colore rosso cupo.

2243 debolissima luce rossastra diffusa.

2300 il fenomeno è completamente scomparso; rimane soltanto la luce della Luna sorta da pochi minuti.

\section{8-9 LUGLIO 1958.}

Il fenomeno veniva notato alle ore 21 (T. U.) quando, essendosi spenta la luce del crepuscolo, stavano per essere iniziate le consuete osservazioni astronomiche.

$21^{\text {h02m }}$ Inizio dell'osservazione. Luminosità generale nella zona Nord. Si notano, particolarmente intensi, due raggi in direzione N-NW di circa $30^{\circ}$ di lunghezza e $3^{\circ}$ di larghezza.

2105 Appaiono, particolarmente intensi, due raggi rosa: uno a Nord e l'altro a $\mathrm{NW}$, che si accentuano sempre più. Il raggio a Nord, successivamente, si allarga e si intensifica, poi si suddivide in tre raggi principali compatti ed uno secondario staccato dal resto di circa $4^{\circ}$ verso Ovest. Il gruppo si intensifica colorandosi sempre più vivamente in rosa. Poco dopo il raggio a NW scompare. Dimensioni del gruppo di raggi Nord: larghezza totale $22^{\circ}$; altezza sull'orizzonte $35^{\circ}$. La zona Nord si colora sempre più 
in rosa mentre torna a comparire anche la luminosità a NW. La luminosità nella zona Nord non è più disposta secondo raggi ma ha un aspetto sfumato ed omogeneo simile, anche nella forma, a quello della luce zodiacale.

$21^{\mathrm{h}} 10^{\mathrm{m}}$ Appare un doppio raggio leggerissimo della lunghezza di c. $30^{\circ}$ e larghezza di c. $12^{\circ}$ a $\mathrm{N}-\mathrm{NW}$.

2112 Scomparsa la luminosità a NW.

2114 Leggera, tenue luminosità generale e due raggi debolissimi. Complessivamente il fenomeno si è notevolmente indebolito.

2116 Si vedono solo tre raggi, sfumatissimi ed appena percettibili, convergenti verso il punto Nord. Poi appaiono due raggi appena visibili: uno in direzione $\mathrm{N}-\mathrm{NW}$, un altro, leggermente più intenso, a NW.

2122 Leggera luminosità generale diffusa in tutta la zona Nord.

2123 Appare a NW un debolissimo raggio lungo circa $22^{\circ}$ e largo circa $4^{\circ}$.

2126 Il raggio appare ora allargato e sfumato mentre si è riformato un raggio di minore intensità a $\mathrm{N}-\mathrm{NW}$. Intensitì dei due raggi: circa come la luce zodiacale normale.

2130 Tutto scomparso, tranne una leggera luminosità appena percettibile a NW.

2133 Si intravedono nuovamente dei raggi disposti secondo un arco centrato a Nord, dell'altezza massima sull'orizzonte di circa $10^{\circ}$ e della lunghezza di circa $90^{\circ}$.

2136 Dal momento di quest'ultima apparizione i raggi delle varie zone dell'arco hanno subito rafforzamenti ed indebolimenti alterni e in questo momento sono piuttosto sfumati.

2138 Appare un raggio in direzione Nord, debole quasi come gli altri, ma lungo circa $45^{\circ}$.

2140 Tutti i raggi sono pressochè scomparsi. Rimangono due leggere luminosità in direzione Nord e $\mathrm{NW}$, la prima intensa come la normale luce zodiacale, la seconda appena percettibile.

2142 Pressochè scomparsa ogni traccia di luminosità.

2148 Appare a Nord una zona (che si indovina formata da raggi) di $20^{\circ}$ di ampiezza e $20^{\circ}$ di altezza. 
$21^{\mathrm{h}} 50^{\mathrm{m}}$ Scomparsa anche questa luminosità.

2203 Nessuna traccia di aurora fino a questo momento in cui tornano a vedersi due leggere luminosità, appena raggiate, in direzione $\mathrm{N}-\mathrm{NW}$ e $\mathrm{NW}$.

2205 Rimane, molto debole, il raggio a N-NW.

2208 Pressochè scomparsa ogni traccia di luminosità tranne una leggerissima luce aurorale in direzione $\mathrm{N}-\mathrm{NW}$.

2217 Si forma a Nord una debole zona luminosa larga circa $50^{\circ}$ ed alta circa $15^{\circ}$.

2222 Scomparsa ogni luminosità.

2228 Continua a non vedersi più nulla, tranne un debole chiarore all'orizzonte intorno al punto Nord.

3210 Non si vede più nulla da parecchio tempo. La Luna è sorta da $45^{\mathrm{m}}$. L'osservazione viene sospesa.

2400 Un ultimo controllo conferma la scomparsa di ogni traccia di luminosità, al di fuori del debole chiarore lunare.

\section{4-5 SETTEMBRE 1958.}

Il fenomeno veniva avvistato a 2045 (T. U.). Si manifestava sotto forma di una luminosità generale diffusa color porpora, nella zona Nord, visibile nonostante il cielo velato o addirittura coperto.

$20^{\mathrm{h}} 50^{\mathrm{m}}$ Appare un raggio alto circa $40^{\circ}$ a $\mathrm{N}-\mathrm{NW}$. La luce diffusa di fondo presenta la massima intensità, con colore sempre rossastro, nella zona N-NE. Poco dopo il raggio tende a sfumare e a confondersi con la zona di fondo.

2052 Il raggio a $\mathrm{N}-\mathrm{NW}$ è pressochè scomparso; è diminuita anche la intensità generale del fenomeno.

2054 Appare in direzione Nord un intenso raggio che presto si trasforma in una zona larga e diffusa mentre l'intensità generale del fenomeno aumenta.

2055 Raggi gialli molto intensi, larghi c. $10^{\circ}$ e alti c. $45^{\circ}$.

2057 Il fenomeno presenta la massima intensità in due zone diffuse a Nord e a Nord-Est, prevalentemente nella prima. Successiva- 
mente, a Nord-Est, appare una serie di raggi, emergenti dalle nuvole, che si intensificano sempre più, giungenti all'altezza della stella Mizar e della larghezza di $10^{\circ}$.

$21^{\mathrm{h}} 00^{\mathrm{m}}$ I raggi sono scomparsi; la luce aurorale generale si è notevolmente attenuata. Dal momento dell'inizio del fenomeno la nebulosità è continuamente diminuita; a questo istante le nubi coprono una fascia di cielo di appena $10^{\circ}$ sull'orizzonte.

2105 Il fenomeno, ridotto ormai ad una leggerissima luminosità generale diffusa appena percettibile, viene ravvivato da un leggero raggio apparso esattamente a Nord che poco dopo sfuma confondendosi con il fondo. E visibile la Luna (in età di giorni 20) all'orizzonte.

2108 La luminosità a Nord, rimasta fino ad ora di intensità costante, sta ora diminuendo ulteriormente. Leggerissima luminosità a NW.

2110 Non si vedono più tracce di luce aurorale.

2112 Torna a comparire, a Nord, una leggera luminosità, larga $20^{\circ}$ e alta $25^{\circ}$, che sparisce però nel minuto successivo. Ogni manifestazione del fenomeno è praticamente scomparsa.

2114 Luminosità raggiata di color rosa scuro presente nella zona Nord ed estendentesi verso NW, larga ed alta $30^{\circ}$. Due raggi spiccano particolarmente.

2117 Compare anche un raggio piuttosto sfumato ad Ovest, che scompare entro i due minuti successivi.

2121 La luminosità a Nord, che si è via via indebolita, è oramai appena percettibile. La luce della Luna comincia a disturbare.

2123 Nessuna traccia di aurora.

2130 Appaiono luci raggiate rossastre nella zona Nord e N-NE. La luce della Luna disturba molto.

2132 Compare a N-NE una zona rossastra raggiata che si intensifica sempre più.

2134 Permane la luminosità rossastra a N-NE ed appare un doppio raggio a NW. 
2135 Il raggio a NW è pressochè scomparso. Tulta la luminosità si è affievolita, traccie sono appena visibili a $\mathrm{N}-\mathrm{NE}$.

2137 Non si vedono più traccie di aurora. Le nuvole sono completamente scomparse fino all'orizzonte. La Luna disturba notevolmente.

2205 Non si vedono più traccie di aurora. Ciò però può essere doruto alla ormai preponderante luce lunare.

2225 A $\mathrm{N}-\mathrm{NW}$, sotto le tre stelle $\varepsilon, \zeta, \eta \mathrm{U}$ Ma, compare una zona rossa piuttosto intensa spostantesi in azimut verso Nord. Raggio molto intenso.

2229 In direzione $\mathrm{N}-\mathrm{NW}$ compare un magnifico raggio, alto circa $45^{\circ}$, e che si intensifica sempre più. Tutta la zona tra Nord e NW, per una lunghezza di c. $30^{\circ}$, è colorata di un rosso intenso. Zona e raggio si intensificano sempre più.

22 32 La luminosità tende a svanire pressochè completamente.

2233 Compare un raggio congiungente le due stelle $\alpha$ e $\beta$ U Ma. Il raggio si rinforza mentre ne compare un altro verso Est e si rinforzano anche quelli a NW. I raggi a NW si rinforzano assumendo un colore giallastro.

2236 I tre raggi cedono il posto a tre zone con luminosità diffusa rossastra. Il raggio giallo a NW è completamente sparito.

2239 Luminosità diffusa larga c. $60^{\circ}$ ed alta c. $30^{\circ}$.

2242 Si intravede una luminosità generale debolissima.

2246 Luce rossastra a NE.

2249 Non si vedono più traccie del fenomeno.

2253 Leggerissima luce rossastra tra Nord e N-NE.

2258 Nessuna traccia del fenomeno.

2315 Nessuna traccia del fenomeno.

2335 Un'ultima occhiata al cielo non rivela alcuna traccia del fenomeno. E però da rilevare che la preponderante luce della Luna rende impossibile l'osservazione di eventuali deboli luminosità. 
RÉSUMÉ RÉIIGÉ SUR LE FORMULAIRE DE THOMSEN 21-22 JANVIER 1957

\begin{tabular}{|c|c|c|c|c|}
\hline $\begin{array}{l}\text { Heure } \\
\text { T. U. }\end{array}$ & Forme & \begin{tabular}{|c|} 
T'en- \\
dence
\end{tabular} & Coordumnées & Remarques \\
\hline 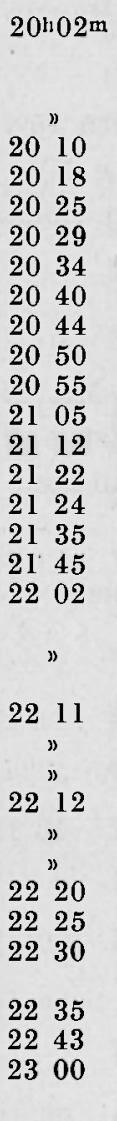 & $\begin{array}{l}\mathrm{R} \\
\mathrm{G} \\
2 \mathrm{R} \\
\mathrm{G} \\
\mathrm{D} \\
\mathrm{G} \\
\mathrm{R} \\
\mathrm{R} \\
\mathrm{G} \\
\mathrm{G} \\
\mathrm{G} \\
\mathrm{D} \\
\mathrm{D} \\
\mathrm{G} \\
\mathrm{RA} \\
\mathrm{G} \\
\mathrm{RA} \\
\mathrm{G} \\
\mathrm{R} \\
\mathrm{RA} \\
\mathrm{G} \\
\mathrm{R} \\
\mathrm{RA} \\
\mathrm{D} \\
2 \mathrm{D} \\
2 \mathrm{D} \\
\mathrm{S}\end{array}$ & 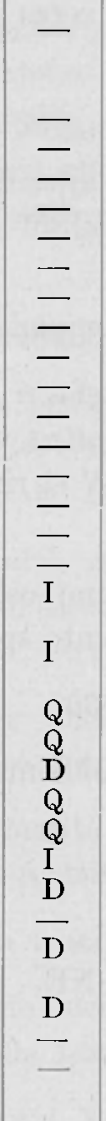 & $\begin{array}{c}295 /-, 340 /- \\
- \\
345 /-, 15 /- \\
-/ 20,-/ 20, \\
320 /-, 340 /-, \\
320 /-, 340 /-, \\
\overline{0} \\
0(\text { centre }) \\
0(\text { centre }) \\
0(\text { centre }) \\
320 /-, 340 /-, \\
335 /-, 20 /- \\
340(\text { centre }) \\
340(\text { centre }) \\
340(\text { centre }) \\
300 / 0,0 / 60,60 / 0 \\
0(\text { centre }) / 15 \\
" \\
" \\
\text { - } \\
\text { " } \\
\text { - } \\
340 \text { (centre)/20 } \\
310(\text { centre }) / 20 \\
10(\text { centre }) / 20 \\
\text { - } \\
-\end{array}$ & $\begin{array}{l}\text { Ciel clair; début des observa- } \\
\text { tions; rougeâtre, estompé } \\
\text { rose et verte vers le Nord } \\
\text { Jaune } \\
\text { Absence d'aurore } \\
\text { Intense } \\
\text { Rougeâtre } \\
\text { " } \\
\text { Absence d'aurore } \\
\text { Apparition e dispar. rapide } \\
\text { " très faible } \\
\text { " plusieurs rais faibles } \\
\text { Rougeâtre } \\
\text { Rose } \\
\text { Rouge faible } \\
\text { Intense } \\
\text { Faible } \\
\text { Rougeâtre } \\
\text { R jaunes, estompés vert; fond: } \\
\text { arc rose-rouge de } 25^{\circ} \text { de larg. } \\
\text { Au dessous de l'are: blanche- } \\
\text { verdâtre très intense } \\
\text { " } \\
\text { " } \\
\text { " } \\
\text { " } \\
\text { Rouge sombre } \\
\text { Rouge sombre } \\
\text { Re sombre, faibles } \\
\text { Rouge someâtre } \\
\text { Absence d'aurore, lueur de la } \\
\text { Lune fin des observations }\end{array}$ \\
\hline
\end{tabular}


8-9 JUILLET 1958

\begin{tabular}{|c|c|c|c|c|}
\hline $\begin{array}{l}\text { Heure } \\
\text { T. U. }\end{array}$ & Forme & $\begin{array}{l}\text { Ten- } \\
\text { dence }\end{array}$ & Coordonnées & Remarques \\
\hline $21^{\mathrm{h}} 02^{\mathrm{m}}$ & $G$ & 一 & $0 /-$ & Début des observations \\
\hline$"$ & $2 \mathrm{R}$ & - & $340 / 30$ & - \\
\hline 2105 & $2 \mathrm{R}$ & $\mathrm{I}$ & $315 /-, 0 /-$, & Rose \\
\hline 2109 & $\mathrm{D}$ & I & 0 (centre) $/ 35$ & $"$ \\
\hline ” & $\mathbf{R}$ & $\mathrm{D}$ & $315 /-$ & - \\
\hline ” & $\mathrm{S}$ & Q & $\begin{array}{l}340 \text { (centre }) /- \\
0(\text { centre }) / 20\end{array}$ & Rose \\
\hline 2110 & $\mathbf{R}$ & - & $340 / 30$ & Très legère \\
\hline 2114 & $\mathrm{G}$ & - & - & Très faible \\
\hline 2116 & $\mathbf{R}$ & - & - & se \\
\hline 2122 & G & - & 0 (centre $) /-$ & $"$ \\
\hline 2123 & $\mathrm{R}$ & - & $315 / 20$ & $"$ \\
\hline 2126 & $2 \mathrm{R}$ & - & $315 /-, 340 /-$ & Faible \\
\hline 2130 & - & - & - & Absence d'aurore \\
\hline 2133 & RA & - & $330 / 0,0 / 10,30 / 0$ & Faible \\
\hline 2138 & $\mathrm{R}$ & - & $0 / 45$ & $"$ \\
\hline 2140 & $2 \mathrm{~S}$ & - & $\begin{array}{l}315 \text { (centre) } /- \\
0(\text { centre }) /-\end{array}$ & - \\
\hline 2142 & 一 & - & - & Absence d'aurore \\
\hline 2148 & $\mathrm{~S}$ & - & 0 (centre) $/ 20$ & Faible \\
\hline 2150 & - & - & - & Absence d'aurore \\
\hline 2203 & $2 \mathrm{D}$ & 一 & $\begin{array}{l}315 \text { (centre) } /- \\
340(\text { centre }) /-\end{array}$ & Faibles \\
\hline 2205 & D & - & 340 (centre) $)-$ & Très faibles \\
\hline 2208 & - & - & - & Absence d'aurore \\
\hline 2217 & G & - & $335 / 0,0 / 15,25 / 0$ & Faible \\
\hline $\begin{array}{ll}22 & 22 \\
24 & 00\end{array}$ & - & - & - & $\begin{array}{l}\text { Absence d'aurore; lueur de la } \\
\text { Lune; fin des observations }\end{array}$ \\
\hline
\end{tabular}




\begin{tabular}{|c|c|c|c|c|}
\hline $\begin{array}{l}\text { Heure } \\
\text { T. U. }\end{array}$ & Forme & $\begin{array}{l}\text { Ten- } \\
\text { dence }\end{array}$ & Conrdunnées & Remarques \\
\hline $20^{\mathrm{h}} 45^{\mathrm{m}}$ & G & - & $0($ centre $) /-$ & $\begin{array}{l}\text { Début des observations ciel } \\
\text { voile ou couvert }\end{array}$ \\
\hline 2050 & $\mathrm{R}$ & - & $340 / 40$ & - \\
\hline 20 & G & $\overline{\mathrm{D}}$ & $20($ centre $) /-$ & Rougeâtre \\
\hline 2054 & $\mathrm{R}$ & I;RS & $\overline{0}$ & E \\
\hline 2055 & $\mathrm{R}$ & - & - & $\begin{array}{l}\text { Jaunes, très intenses larg. } 10^{\circ} \text {, } \\
\text { haut. } 45^{\circ}\end{array}$ \\
\hline 2057 & $\mathrm{~S}$ & - & $0 /-$ & - \\
\hline $20 " 58$ & $\begin{array}{l}S \\
R\end{array}$ & $\bar{I}$ & $\begin{array}{l}45 /- \\
45 / 15\end{array}$ & - \\
\hline 2100 & G & $\mathrm{D}$ & (5) & $\begin{array}{l}\text { Le ciel est toujours plus clair; } \\
\text { les nuages couvrent à ce } \\
\text { moment une bande de } 10^{\circ} \\
\text { de hauteur }\end{array}$ \\
\hline 2105 & G & Q & 0 (centre) $/-$ & $\begin{array}{l}\text { Très faible: Lune visible à } \\
\text { l'horizont }\end{array}$ \\
\hline 2108 & G & $\mathrm{D}$ & $0($ centre $) /-$ & $-\infty$ \\
\hline $\begin{array}{ll}21 & 10 \\
21 & 12\end{array}$ & $\mathrm{~s}$ & $\bar{I}$ & $\overline{0(\text { centre }) / 25}$ & $\begin{array}{l}\text { Absence d'aurore } \\
\text { Faible }\end{array}$ \\
\hline 2113 & S & $\mathrm{D}$ & & (1) \\
\hline 2114 & D & - & $330 /-, 0 / 30$ & Rose sombre \\
\hline 2117 & $\mathrm{R}$ & - & $300 /-$ & 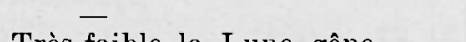 \\
\hline $\begin{array}{ll}21 & 21 \\
21 & 23\end{array}$ & S & 二 & $0 /-$ & $\begin{array}{l}\text { Très faible la Lune gêne } \\
\text { Absence d'aurore }\end{array}$ \\
\hline 2130 & D & - & $0 /-, 45 /-$ & $\begin{array}{l}\text { Rougeâtre; la Lune gêne beau- } \\
\text { coup }\end{array}$ \\
\hline $\begin{array}{ll}21 & 32 \\
21 & 34\end{array}$ & $\begin{array}{l}\mathrm{D} \\
\mathrm{D}\end{array}$ & $\begin{array}{l}\mathrm{I} \\
\mathrm{Q}\end{array}$ & $\begin{array}{l}20 \prime- \\
20 /-\end{array}$ & Rougeâtre \\
\hline$"$ & $\mathrm{R}$ & - & $315 /-$ & - \\
\hline $\begin{array}{l}2135 \\
2137\end{array}$ & G & 二 & - & $\begin{array}{l}\text { Faible } \\
\text { Absence d'aurore; ciel clair; } \\
\text { la Lune gêne beaucoup }\end{array}$ \\
\hline 2205 & — & - & - & $\begin{array}{l}\text { Absence d'aurore; la lueur de } \\
\text { la Lune est prépondérante }\end{array}$ \\
\hline 2225 & $\begin{array}{l}\mathrm{S} \\
\mathrm{R}\end{array}$ & WN & 二 & Rouge \\
\hline 2229 & $\begin{array}{l}\mathrm{K} \\
\mathrm{R}\end{array}$ & $\overline{\mathrm{I}}$ & $34 \overline{0 / 45}$ & Très fort \\
\hline $222^{\prime \prime} 32$ & $\begin{array}{l}\mathrm{G} \\
\mathrm{G}\end{array}$ & $\begin{array}{l}\text { I } \\
\text { D }\end{array}$ & $\begin{array}{l}330 /-, 0 /- \\
n\end{array}$ & Rouge vif \\
\hline 2233 & $\mathrm{R}$ & I & $340 /-, 350 / 15,20 /-$ & Jaune \\
\hline 2236 & $S$ & - & " & Rougeâtre \\
\hline $\begin{array}{l}2239 \\
2242\end{array}$ & G & - & ${ }^{330 /-, 0 / 30,30 /-}$ & $\begin{array}{l}\text { Lueur gènerale (à Nord) très } \\
\text { faible }\end{array}$ \\
\hline 2246 & G & - & $45 /-$ & Rougeâtre \\
\hline 2249 & - & - & -1 & Absence d'aurore \\
\hline 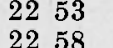 & G & 二 & $10 /-$ & Rougeâtre, très faible \\
\hline $\begin{array}{l}2258 \\
2315\end{array}$ & - & 二 & - & Absence d'aurore \\
\hline 2335 & - & - & - & $\begin{array}{l}\text { Absence d'aurore; la Lune est } \\
\text { très haute et la continuation } \\
\text { des observations n'est plus } \\
\text { possible; fin des observations }\end{array}$ \\
\hline
\end{tabular}




\section{RIASSUNTO}

Vengono descritte tre aurore boreali osservate dalla Stazione Astronomica di Loiano (Bologna), le notti del: 21-22 Gennaio 1957; 8-9 Luglio 1958; 4-5 Settembre 1958.

\section{$A B S T R A C T$}

Three auroras observed from the :Astronomical Observatory of Loiano (Bologna) in the nights of 21-22 January 1957; 8-9 July 1958; 4-5 September 1958, are described.

\section{BIBLIOGRAFIA}

Ciel et Terre, 73, 527 e 546; 74, 48. 